\title{
Adeno-Associated Virus-Mediated Mini-Agrin Delivery Is Unable to Rescue Disease Phenotype in a Mouse Model of Limb Girdle Muscular Dystrophy Type 2I
}

\author{
Charles H. Vannoy, ${ }^{*}$ Haowen Zhou, ${ }^{\dagger}$ Chunping Qiao, ${ }^{\ddagger}$ Xiao Xiao, ${ }^{\ddagger}$ Anne G. Bang, ${ }^{\dagger}$ and Qi L. Lu*
}

\begin{abstract}
From the McColl-Lockwood Laboratory for Muscular Dystrophy Research,* Cannon Research Center, Carolinas Medical Center, Carolinas Healthcare System, Charlotte, North Carolina; the Conrad Prebys Center for Chemical Genomics, ${ }^{\dagger}$ Sanford Burnham Prebys Medical Discovery Institute, La Jolla, California; and the Division of Molecular Pharmaceutics, ${ }^{\ddagger}$ Eshelman School of Pharmacy, University of North Carolina at Chapel Hill, Chapel Hill, North Carolina
\end{abstract}

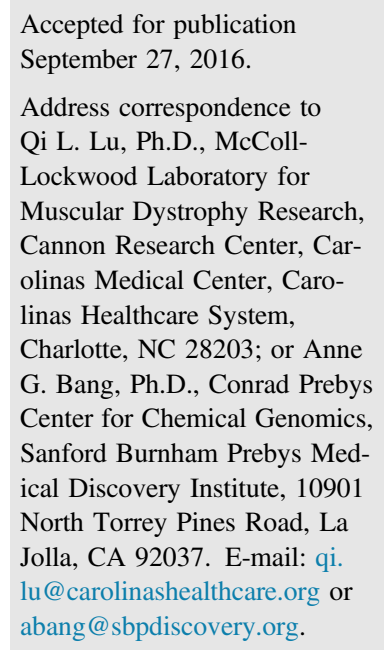

\begin{abstract}
Agrin is a basement membrane-specific proteoglycan that can regulate orientation of cytoskeleton proteins and improve function of dystrophic skeletal muscle. In skeletal muscle, agrin binds with high affinity to laminin(s) and $\alpha$-dystroglycan $(\alpha-D G)$, an integral part of the dystrophin-glycoprotein complex. Miniaturized forms of agrin (mAgrin) have been shown to ameliorate disease pathology in a laminin- $\alpha 2$ knockout mouse model of muscular dystrophy, acting as a link between $\alpha-D G$ and laminin(s). Here, we test whether mAgrin might also improve pathologies associated with FKRP-related dystroglycanopathies, another form of muscular dystrophy characterized by weak interactions between muscle and basement membranes. We demonstrate in vitro that mAgrin enhances laminin binding to primary myoblasts and fibroblasts from an FKRP mutant mouse model and that this enhancement is abrogated when mAgrin is in molar excess relative to laminin. However, in vivo delivery of mAgrin via adeno-associated virus (AAV) into FKRP mutant mice was unable to improve dystrophic phenotypes, both histologically and functionally. These results likely reflect insufficient binding of mAgrin to hypoglycosylated $\alpha$-DG on muscle fibers and possibly abrogation of binding from molar excess of overexpressed AAV-delivered mAgrin. Further exploration of mAgrin modification is necessary to strengthen its binding to other membrane components, including hypoglycosylated $\alpha-D G$, for potential therapeutic applications. (Am J Pathol 2017, 187: 431-440; http://dx.doi.org/ 10.1016/j.ajpath.2016.09.024)
\end{abstract}

Limb-girdle muscular dystrophies (LGMDs) are a genetically heterogeneous group of rare disorders associated with progressive wasting and weakness of the skeletal muscles. Currently, there are $>30$ different subtypes of LGMD, each of which has been linked to specific gene loci, that reveal a variable phenotypic spectrum. ${ }^{1}$ One gene in particular, $F K R P$, has been known to exhibit mutations that disrupt post-translational modification and subsequently result in the abnormal glycosylation of $\alpha$-dystroglycan ( $\alpha$-DG). $F K R P$-related dystroglycanopathies are associated with a wide spectrum of clinical severities that include LGMD type 2I (LGMD2I), the most common, yet milder form. ${ }^{2,3}$ This form of dystroglycanopathy displays a characteristic feature of insufficient $O$-linked mannosyl glycosylation of $\alpha$-DG, a modification which is essential for protein-protein interactions within the extracellular matrix, specifically the binding of $\alpha$-DG to laminin and other extracellular matrix proteins (ie, agrin, perlecan, neurexin, and biglycan). ${ }^{4,5}$ As a result, the skeletal muscle fiber membrane integrity is compromised, resulting in muscle fiber damage and degeneration.

With the use of knock-in technology, we have genetically engineered an LGMD2I mouse model associated with an

Supported by the Carolinas Muscular Dystrophy Research Endowment at the Carolinas HealthCare Foundation (C.H.V. and Q.L.L.) and the LGMD2I Research Fund grant CHF2147 (X.X., A.G.B., and Q.L.L.).

Disclosures: None declared. 
FKRP mutation that results in an amino acid change from proline-to-leucine at position 448 (FKRP ${ }^{\mathrm{P} 448 \mathrm{~L}}$ mutant). ${ }^{6}$ The FKRP ${ }^{\mathrm{P} 448 \mathrm{~L}}$ mutant mouse currently represents one of the few viable, genetically engineered models that displays a muscle pathologic process similar to that observed in LGMD2I patients with a moderately severe dystrophic phenotype void of evident brain or eye anomalies. Other examples include the $\mathrm{L}_{276 \mathrm{I}^{\mathrm{KI}}}$ and $\mathrm{FKRP}_{\mathrm{MD}}$ mouse models. $^{7,8}$ Within 2 to 3 weeks of age, the FKRP $^{\mathrm{P} 448 \mathrm{~L}}$ mutant mouse exhibits a clear dystrophic phenotype with variation in fiber size distribution, central nucleation of fibers, and a population of necrotic fibers. ${ }^{7}$ As the mice age, fibrosis, fat infiltration, and an increase in collagen deposition within interstitial space becomes clearly discernible. These dystrophic characteristics are observed in all skeletal muscle groups, including the diaphragm, and, to some degree, show evidence of cardiac involvement.

Agrin is a highly glycosylated heparan sulfate proteoglycan that exists as several isoforms in various tissue types. ${ }^{9}$ The neural isoform of agrin has long been considered the main organizer for postsynaptic differentiation at the neuromuscular junction; however, it is the muscle isoform that has garnered much attention for its ability to regulate the orientation of cytoskeleton proteins and to ameliorate function in dystrophic skeletal muscle. ${ }^{10-12}$ The chemical nature of agrin allows for its amino-terminal domain to bind to a number of laminin variants ${ }^{13-15}$ and its carboxy-terminal domain to bind to $\alpha$-DG. ${ }^{16-18}$ Therefore, agrin is considered a bifunctional linker molecule. Exploring its potential of multiple ligand binding capacity, Rüegg and colleagues ${ }^{12,19}$ were the first to our knowledge to demonstrate that overexpressing a miniaturized form of agrin (mAgrin) derived from the muscle isoform ameliorated disease pathologic progression in a laminin- $\alpha 2$ knockout mouse model of congenital muscular dystrophy. This initial study was accomplished by breeding chick mAgrin transgenic mice with mice carrying a null mutation in the LAMA2 gene. However, the transgenic approach is not clinically applicable and is complicated by the mAgrin overexpression during the early stage of muscle and nerve development. Consequently, Qiao et $\mathrm{al}^{20}$ conducted an adeno-associated virus (AAV)-mediated gene therapy approach to assess the therapeutic potential of mouse mAgrin in the treatment of mice with $L A M A 2$ deficiencies. In their study, they demonstrated that overexpression of the mAgrin ameliorated muscle structural characteristics, decreased fibrosis, and restored the structural integrity of the basal lamina of muscle myofibers.

Herein, we examined the therapeutic potential of mouse mAgrin expression to $F K R P$-related dystroglycanopathy via AAV-mediated gene therapy. Specifically, we aimed to understand whether expression of mAgrin is able to enhance the binding of laminin(s) to hypoglycosylated $\alpha$-DG and to ameliorate the dystrophic phenotypes caused by the hypoglycosylation of $\alpha$-DG. First, we demonstrate in a cell-based assay that purified recombinant mAgrin ${ }^{21}$ enhances laminin binding to primary myoblasts and fibroblasts from FKRP $^{\mathrm{P} 448 \mathrm{~L}}$ mutant and $\mathrm{C} 57 \mathrm{BL} / 6$ mice and that this enhancement is abrogated when mAgrin is in molar excess to laminin. Next, we delivered mAgrin via AAV into FKRP $^{\mathrm{P} 448 \mathrm{~L}}$ mutant mice. Our results reveal that mAgrin is unable to improve dystrophic phenotypes, histologically and functionally, supporting evidence that binding of agrin to $\alpha$-DG critically depends on functional glycosylation of $\alpha-D G$ and linkage of agrin to extracellular matrix proteins. Nevertheless, mAgrin demonstrates a high affinity for basement membrane components in the absence of glycosylated $\alpha$-DG, motivating further exploration to strengthen its binding to other membrane components, including hypoglycosylated $\alpha-D G$, for therapeutic applications.

\section{Materials and Methods}

\section{Mouse Model}

FKRP $^{\mathrm{P} 448 \mathrm{~L}}$ mutant mice were generated by inGenious Targeting Laboratory (Stony Brook, NY). The targeting vector was engineered with a c.1343C $>\mathrm{T}$ point mutation in exon 3 of the mouse FKRP gene, resulting in an amino acid change from proline-to-leucine at position $448 .{ }^{6}$ A loxP/FRT-flanked neomycin phosphotransferase gene expression cassette was inserted in intron 2 and subsequently removed in vivo by crossbreeding with C57BL/6 Flp mice and further backcrossed to C57BL/6 for 10 generations by inGenious Targeting Laboratory to generate the homozygote FKRP $^{\mathrm{P} 448 \mathrm{~L}}$ mutant mice (Supplemental Figure S1). Colonies of C57BL/6 mice were originally obtained from The Jackson Laboratory (Bar Harbor, ME) and used as controls. All mice were housed in the vivarium of Carolinas Medical Center according to animal care guidelines of the institute. All animal studies were approved by the Institutional Animal Care and Use Committee of Carolinas Medical Center.

\section{Mouse Myoblast/Fibroblast Culture Generation}

Primary mouse skeletal muscles (quadriceps) were isolated from 3-week-old $\mathrm{FKRP}^{\mathrm{P} 448 \mathrm{~L}}$ mutant and $\mathrm{C} 57 \mathrm{BL} / 6$ mice. Muscles were placed in a washing medium $[1 \%$ penicillinstreptomycin, 1\% Fungizone (Thermo Fisher Scientific, Waltham, MA) in Dulbecco's modified Eagle's medium] and washed multiple times before isolation. The muscle was then minced into fine pieces and placed in a warmed solution containing collagenase $(1 \mathrm{mg} / \mathrm{mL})$ in Ham's F10 medium and shaken for 3 to 4 hours in an incubator at $37^{\circ} \mathrm{C}$. The cells were then centrifuged for 5 minutes at $524 \times g$ at $4^{\circ} \mathrm{C}$. The pellet was then resuspended in growth medium (20\% fetal bovine serum, $2 \%$ L-glutamine, and $1 \%$ penicillin-streptomycin in Ham's F10 medium) and centrifuged again for 5 minutes at $524 \times g$ at $4^{\circ} \mathrm{C}$. The cells were resuspended in growth medium and plated onto collagencoated T25 flasks to allow proliferation of skeletal 
myoblasts and fibroblasts. The growth medium was changed every 5 days until the contents of the flask were $75 \%$ confluent, approximately 1 to 2 weeks. Once $75 \%$ confluent, mouse skeletal myoblasts and fibroblasts were split into dishes for expansion. After approximately 1 week, when the cells were $85 \%$ to $90 \%$ confluent, they were trypsinized and frozen in liquid nitrogen.

\section{Laminin/mAgrin Binding Assay in Vitro}

Primary myoblasts and fibroblasts from $\mathrm{FKRP}^{\mathrm{P} 448 \mathrm{~L}}$ mutant and C57BL/6 mice were plated on 384-well imaging plates. Detection of laminin binding to myoblasts and fibroblasts by immunofluorescence was performed as described previously. ${ }^{22}$ Myoblasts and fibroblasts were exposed to varying ratios of laminin 111 from Engelbreth-Holm-Swarm murine sarcoma basement membrane (Sigma-Aldrich, St. Louis, MO) and purified recombinant chick mAgrin ${ }^{21}$ for 1 hour at $37^{\circ} \mathrm{C}$, followed by fixation in $4 \%$ paraformaldehyde, and detection with polyclonal rabbit anti-laminin (SigmaAldrich) at a dilution of 1:200, and donkey anti-mouse IgG $(\mathrm{H}+\mathrm{L})$ Alexa Fluor 488 (Invitrogen, Carlsbad, CA) at a dilution of 1:500. Images were acquired on an Opera Phenix High Content Screening System (PerkinElmer, Waltham, MA). All data are presented as means \pm SEM.

\section{Construction of AAV Vectors and Administration}

The recombinant AAV vectors (serotype 6) expressing mAgrin (AAV-mAgrin) and enhanced green fluorescent protein (AAV-EGFP) driven by a chicken $\beta$-actin promoter were constructed as described previously (Supplemental Figure S2). ${ }^{23}$ The recombinant AAV vectors were produced and purified using the three-plasmid co-transfection method as previously described. ${ }^{24}$ The viral particles were purified twice through cesium chloride density gradient ultracentrifugation by using the previously published protocol. ${ }^{25}$ Titers of viral particle numbers were determined by the viral DNA dot blot method. Enriched $\left(5 \times 10^{12}\right.$ vector genomes $/ \mathrm{mL}$ ) preparations of $\mathrm{AAV}$-mAgrin and AAV-EGFP were stored in sterile phosphate-buffered saline at $-80^{\circ} \mathrm{C}$ until future use.

The tibialis anterior, quadriceps, and gastrocnemius muscles on the left side of 5-week-old FKRP ${ }^{\mathrm{P} 44 \mathrm{~L}}$ mutant $(n=10$; 5 male and 5 female) mice were injected with $5 \times 10^{12}$ vector genomes $/ \mathrm{mL}$ of AAV-mAgrin. Tibialis anterior muscles were injected with a volume of $40 \mu \mathrm{L}$ using a U-100 insulin syringe, whereas gastrocnemius and quadriceps muscles were injected with a volume of $50 \mu \mathrm{L}$. Injections were always at the belly site of the muscle. Contralateral muscles (right side) were injected with sterile saline $(0.9 \%)$. AAV-EGFP $\left(5 \times 10^{12}\right.$ vector genomes $/ \mathrm{mL}$ ) was used as a control to test the effects of AAV infection and nonspecific gene expression in $\mathrm{FKRP}^{\mathrm{P} 448 \mathrm{~L}}$ mutant $(n=10 ; 5$ male and 5 female) mice. Untreated $\operatorname{FKRP}^{\mathrm{P} 448 \mathrm{~L}}$ mutant $(n=10 ; 5$ male and 5 female) and C57BL/ $6(n=8 ; 4$ male and 4 female) mice were also used as controls. All mice were sacrificed at 14 weeks of age ( 9 weeks after injection for treated mice).

\section{Muscle Histology and Morphometric Analysis}

Tissues were dissected and snap-frozen in dry ice-chilled 2 -methylbutane. Tissues were cryosectioned (6 $\mu \mathrm{m}$ thick), positioned on glass microscope slides, and then stored at $-80^{\circ} \mathrm{C}$ until future use. Frozen tissue sections were stained with hematoxylin and eosin. Muscle cross-sectional fiber radii and percentage of myofibers with centrally located nuclei were determined from tibialis anterior, quadriceps, and gastrocnemius muscles stained with hematoxylin and eosin. Five representative images per section per animal were used. Images were visualized using an Olympus BX51/BX52 fluorescence microscope (Opelco, Dulles, VA) and captured using the Olympus DP70 Digital Camera System (Opelco). All measurements and calculations were conducted using MetaMorph Microscopy Automation \& Image Analysis Software version 7.7.0.0 (Molecular Devices, Sunnyvale, CA). All data are presented as means \pm SEM. Multiple $t$-tests were performed using GraphPad Prism software version 7.00 for Windows (GraphPad Software, La Jolla, CA).

\section{Immunohistochemical and Western Blot Analyses}

Polyclonal antibody against agrin was purchased from R\&D Systems (Minneapolis, MN). Polyclonal antibodies against actin and laminin were purchased from SigmaAldrich. Monoclonal antibody against laminin $\alpha 2$ was purchased from Enzo (Farmingdale, NY). Polyclonal antibody against laminin $\alpha 4$ was purchased from Thermo Fisher Scientific. Polyclonal antibody against laminin $\alpha 5$ was purchased from Santa Cruz Biotechnology (Dallas, TX). For the immunohistochemical detection, frozen tissue sections were incubated with $1 \times$ tris-buffered saline (TBS) for 5 minutes and then immediately blocked with $10 \%$ normal donkey serum or $20 \%$ fetal bovine serum/ $10 \%$ normal goat serum diluted in $1 \times$ TBS for 30 minutes. Sections were then incubated overnight at $4{ }^{\circ} \mathrm{C}$ with primary antibodies [agrin (dilution 1:1000 or 1:100), laminin (dilution 1:200), laminin $\alpha 2$ (dilution 1:200), laminin $\alpha 4$ (dilution 1:50), laminin $\alpha 5$ (dilution 1:50)] diluted in $1 \times$ TBS. Sections were washed two times with $1 \times$ TBS, and appropriate secondary antibodies were incubated at room temperature for 1 hour. Sections were washed three times with $1 \times$ TBS and mounted with Fluorescence Mounting Medium (Dako, Carpinteria, CA) containing 1× DAPI. Images were visualized using an Olympus BX51/BX52 fluorescence microscope (Opelco) and captured using the Olympus DP70 Digital Camera System (Opelco).

For Western blot analysis, tissues were rapidly homogenized in extraction buffer $(50 \mathrm{mmol} / \mathrm{L}$ Tris- $\mathrm{HCl}$, $\mathrm{pH} 8.0,150 \mathrm{mmol} / \mathrm{L} \mathrm{NaCl}, 1 \% \mathrm{SDS}, 1 \%$ Triton X-100) supplemented with $1 \times$ Protease Inhibitor Cocktail (Sigma- 
Aldrich). After protein denaturation $\left(85^{\circ} \mathrm{C}\right.$ for 2 minutes $)$, nondissolved protein was removed by centrifugation $\left(14,000 \times g\right.$ for 15 minutes at $\left.4^{\circ} \mathrm{C}\right)$. Protein concentrations were measured using a NanoDrop 2000 Spectrophotometer (Thermo Fisher Scientific). For each lane, $40 \mu \mathrm{g}$ of protein was loaded, separated on a $4 \%$ to $20 \%$ TrisGlycine polyacrylamide gel (Life Technologies, Carlsbad, CA), and immunoblotted. Nitrocellulose membranes were blocked with Protein-Free T20 (TBS) Blocking Buffer (Thermo Fisher Scientific) for 1 hour at room temperature and then incubated with primary antibodies [agrin (dilution 1:4000) or actin (dilution 1:4000)] overnight at $4^{\circ} \mathrm{C}$. Anti-goat or anti-rabbit horseradish peroxidase-conjugated secondary antibodies were applied to the membranes at a 1:4000 dilution for 1 hour. All blots were developed by electrochemiluminescence immunodetection (PerkinElmer), exposed to BioMax Light film (Sigma-Aldrich), and processed by a Mini-Medical imaging system (AFP Imaging, Mount Kisco, NY).

\section{Creatine Kinase Assay}

Blood was collected by cardiac puncture, and the plasma was separated by centrifugation and frozen at $-20^{\circ} \mathrm{C}$ until further analysis. Creatine kinase levels in plasma were quantitated by IDEXX Laboratories, Inc. (Columbia, MO). All data are presented as means \pm SEM. An unpaired $t$-test with Welch's correction was performed using GraphPad Prism software version 7.00 for Windows (GraphPad Software). A $P$ value $<0.05$ was considered as the level of significance.

\section{Results}

\section{Expression of mAgrin in Vitro Enhances Laminin Binding}

In previous studies, genetic and biochemical analyses of $F K R P$-related dystroglycanopathies indicated that residual
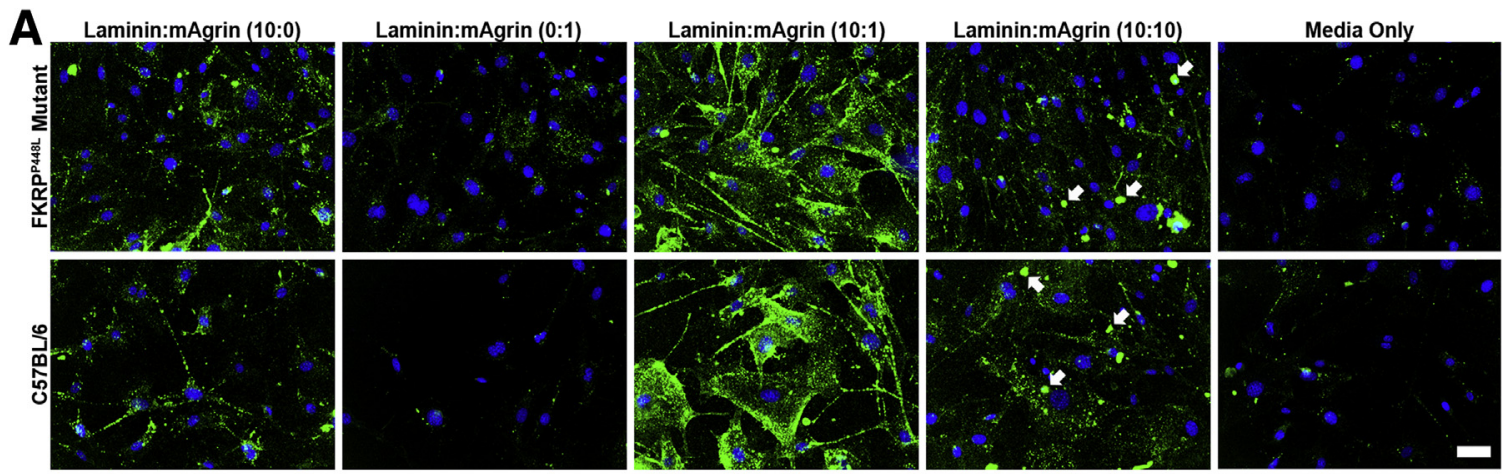

\section{B}

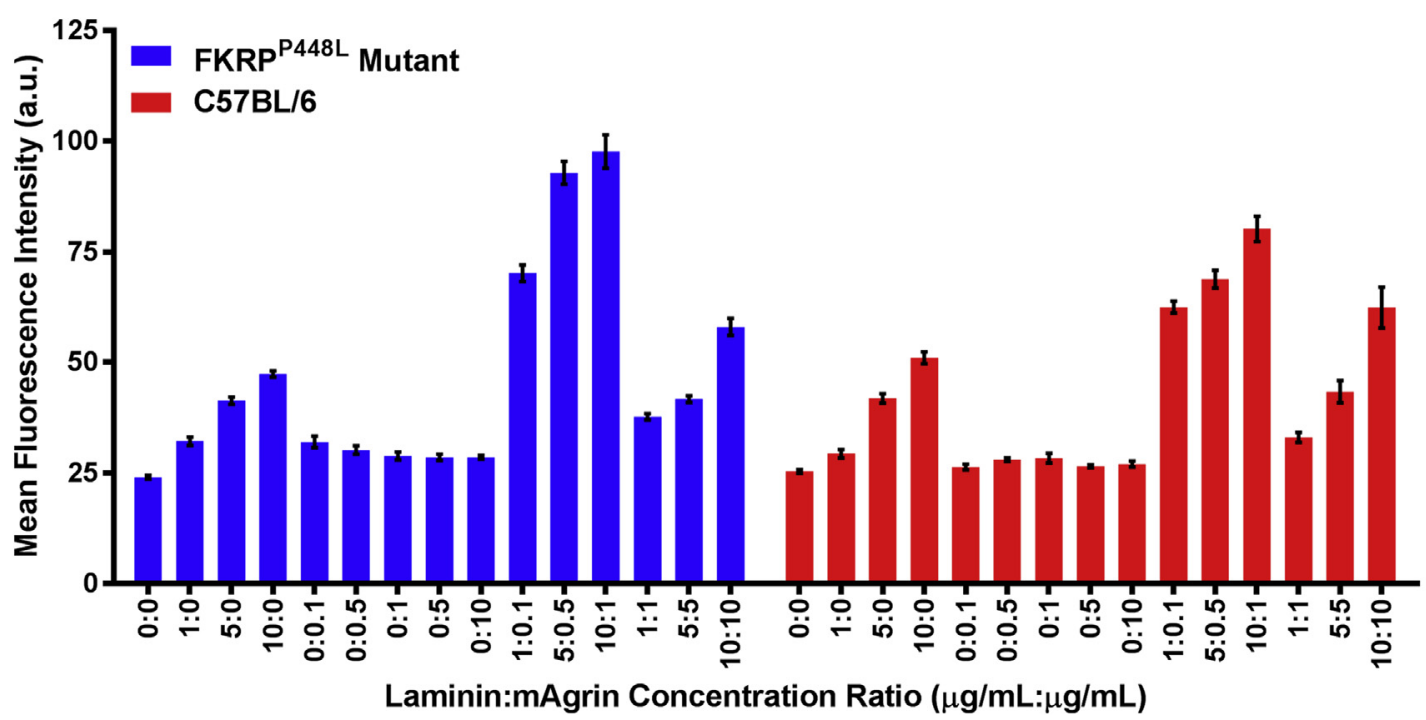

Figure 1 mAgrin enhances binding of laminin to FKRP ${ }^{\mathrm{P} 448 \mathrm{~L}}$ mutant and $\mathrm{C} 57 \mathrm{BL} / 6$ mouse myoblasts and fibroblasts. A: Representative images of laminin binding (green) to FKRP $\mathrm{P}^{\mathrm{P} 48 \mathrm{~L}}$ mutant (top row) and C57BL/6 (bottom row) mouse myoblasts and fibroblasts exposed to indicated concentration ( $\mu \mathrm{g} / \mathrm{mL}$ ) ratios of laminin and mAgrin indicated at the top. Arrows indicate larger laminin-positive aggregates present more frequently when mAgrin is in molar excess to laminin. Cellular nuclei were counterstained with DAPI (blue). B: Quantification of laminin binding signal on FKRP ${ }^{\mathrm{P} 448 \mathrm{~L}}$ mutant (blue bars) and C57BL/6 (red bars) mouse myoblasts and fibroblasts exposed to indicated concentration $(\mu \mathrm{g} / \mathrm{mL})$ ratios of laminin and $\mathrm{mAgrin}$. Error bars represent SD of mean whole cell fluorescence intensity of 6 wells per condition, 100 to 200 cells per 12 fields acquired per well. Scale bar $=50 \mu \mathrm{m}(\mathbf{A})$. a.u., arbitrary unit; FKRP $P^{\mathrm{P} 48 \mathrm{~L}}$ mutant, FKRP mutation that results in an amino acid change from proline-to-leucine at position 448; mAgrin, miniaturized form of agrin. 
glycosylation is present in patients as well as in the muscles of our FKRP ${ }^{\mathrm{P} 448 \mathrm{~L}}$ mutant model. ${ }^{6,26}$ Therefore, we investigated whether purified recombinant chick mAgrin could enhance the binding of the residual glycosylated $\alpha$-DG or the hypoglycosylated $\alpha$-DG to laminin(s) or other extracellular matrix proteins. We examined such potential effects in vitro using cultured myoblasts and fibroblasts from both the $\mathrm{FKRP}^{\mathrm{P} 448 \mathrm{~L}}$ mutant and normal C57BL/6 mice (Figure 1). We observed similar basal levels of laminin binding to both the FKRP ${ }^{\mathrm{P} 448 \mathrm{~L}}$ and C57BL/6 myoblasts and fibroblasts, which was increased upon addition of chick mAgrin in equimolar quantities to laminin (Figure 1A). Furthermore, we observed that a molar excess of chick mAgrin abrogated the enhanced laminin binding in the myoblasts and fibroblasts, a trend that was demonstrated at multiple doses (Figure 1B). Under conditions of excess molar chick mAgrin, we also observed an increase in the formation of larger laminin-positive aggregates, apparently bound to cells (Figure 1A). However, the overall signal for laminin binding was clearly lower than that when equal molar chick mAgrin was used. These results suggest that chick mAgrin has the potential to enhance the link between laminin(s) and membrane of muscle cells with FKRP mutations and that the molar ratio of chick mAgrin to laminin is important.

\section{Construction of Mouse mAgrin}

For in vivo experiments, we used mouse mAgrin to avoid any potential host immune responses to the commonly used chick mAgrin. However, the published mouse agrin $\mathrm{N}$-terminal coding sequence lacks a definitive signal peptide for efficient secretion. Therefore, the signal peptide sequence was deduced from the mouse agrin genomic DNA. ${ }^{27}$ Similar to the chick mAgrin initially reported by Moll et al, ${ }^{12}$ the mouse mAgrin construct consists of the following structural elements: a signal peptide sequence, the amino-terminal agrin domain that binds to laminin, one follistatin-like domain, and the carboxy-terminal region containing the epidermal growth factor-like and laminin globular domain that bind to $\alpha$-DG (Figure 2). The mouse mAgrin gene was subcloned into the AAV serotype 6 vector under the control of a chicken $\beta$-actin promoter for strong expression in skeletal and cardiac muscle. ${ }^{28}$ The mAgrin is flanked by two inverted terminal repeats that are needed as signals for packaging genome into the viral capsid. A poly(A) tail is added to protect the mRNA molecule from enzymatic degradation in the cytoplasm and to aid in transcription termination, export of the mRNA from the nucleus, and translation.

\section{Expression of mAgrin in Dystrophic Mice}

Next, we tested the functionality of the AAV constructs and the procedure of injection by examining the AAV-EGFP expression in vivo via local intramuscular injection into leg

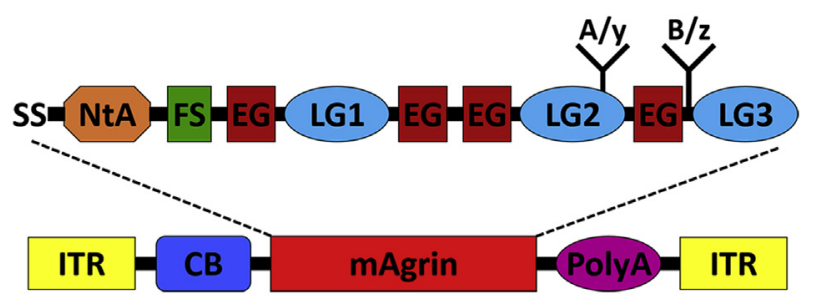

Figure 2 AAV-mAgrin construct. The mAgrin incorporates the following structural elements: a SS, the NtA domain that binds to laminin, one FS, and the carboxy-terminal region containing the EGs and LGs that bind to $\alpha$ $D G$. The mouse mAgrin gene along with a Poly $(A)$ tail was subcloned into the AAV vector under the control of a CB promoter and flanked by two ITRs. $A / y$ and $B / z$ represent alternative splicing sites ( $y$ and $z$ are used to denote these sites in chick and mammals, respectively). AAV, adeno-associated virus; $C B$, chicken $\beta$-actin; $E G$, epidermal growth factor-like domain; $F S$, follistatin-like domain; ITR, inverted terminal repeat; $L G$, laminin globular domain; mAgrin, miniaturized form of agrin; NtA, amino-terminal agrin; SS, signal peptide sequence; $\alpha$-DG, $\alpha$-dystroglycan.

muscles (ie, tibialis anterior, quadriceps, and gastrocnemius) of the $\mathrm{FKRP}^{\mathrm{P} 448 \mathrm{~L}}$ mutant mice. A large number of fibers were identified with strong expression of EGFP fluorescence in all treated muscles with $5 \times 10^{12}$ vector genomes/ $\mathrm{mL}$ (Supplemental Figure S3). We then injected the same amount of AAV-mAgrin into the same muscles of the FKRP $^{\mathrm{P} 448 \mathrm{~L}}$ mutant mice at 5 weeks of age. Nine weeks after injection, we evaluated the therapeutic effects of the mAgrin expression. Immunostaining with the anti-agrin antibody revealed positive signals along the entire circumference of the treated muscle fibers. Expression of mAgrin was detected at high levels in all of the injected muscles (Figure 3A). Conversely, signals for mAgrin were undetectable at the membrane sites of all of the fibers in the contralateral muscles of the treated mice (Supplemental Figure S4). No abnormal signal for mAgrin was detected in the diaphragm, heart, or liver of the treated mice (Supplemental Figure S5). Positive staining was only observed in the blood vessels in these tissues, indicating that the transprotein remained locally after secretion. Similarly, all muscles in the untreated $\mathrm{FKRP}^{\mathrm{P} 448 \mathrm{~L}}$ and $\mathrm{C} 57 \mathrm{BL} / 6$ mice showed only staining in the endomysium, predominantly in the blood vessels. We also measured the expression levels of mAgrin via Western blot analysis (Figure 3B). AAVmAgrin-treated muscle produced positive signals with an expected size of approximately $110 \mathrm{kDa}$. No signal was detected in the untreated $\mathrm{FKRP}^{\mathrm{P} 448 \mathrm{~L}}$ or C57BL/6 muscles, consistent with the fact that agrin expression is restricted to the neuromuscular junction and is insufficient for detection. The equal amount of protein loading was confirmed by the detection of actin for each sample.

To determine the localization of the mAgrin protein more accurately and its relationship with laminin, we conducted a dual staining for the two proteins (Figure 4). Signals for both agrin and laminin superimposed at almost exactly the same site in the AAV-mAgrin-treated muscles, consistent with its binding potential to laminin(s) within the muscle basement membrane. The same staining pattern was observed 
A Tibals Anterior

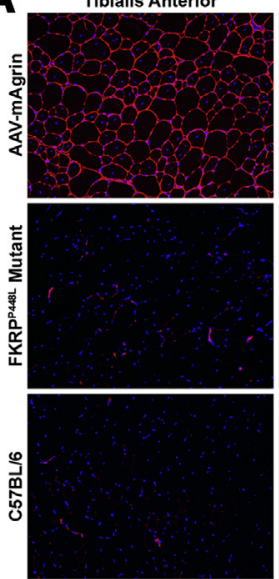

Quadriceps

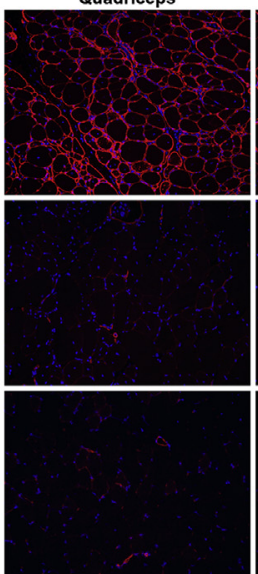

Gastrocnemius

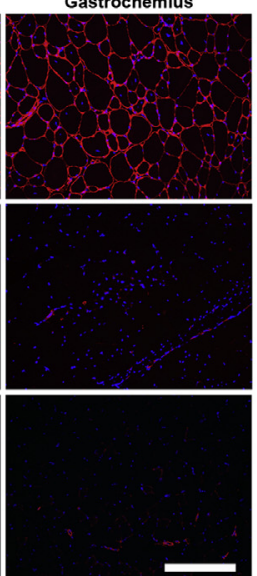

B

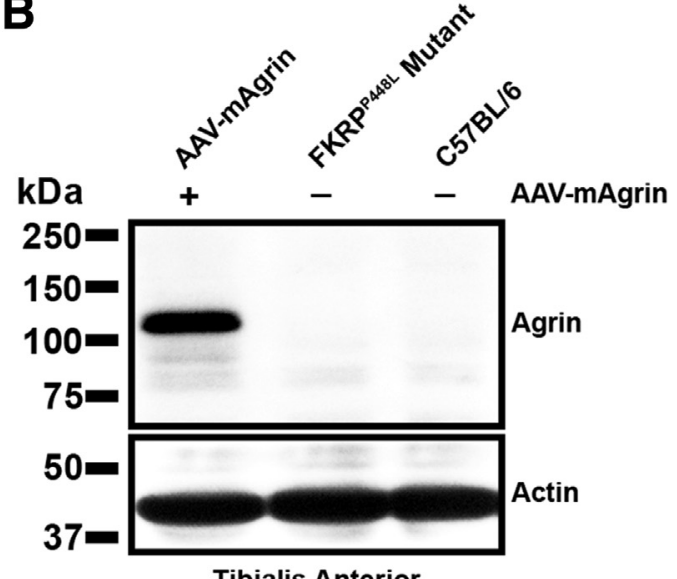

Figure 3 Overexpression of mAgrin in skeletal muscle after AAV-mAgrin gene delivery. A: Immunohistochemical agrin staining (red) of the tibialis anterior, quadriceps, and gastrocnemius muscles from AAV-mAgrin-treated, untreated FKRP ${ }^{\mathrm{P} 448 \mathrm{~L}}$ mutant, and $\mathrm{C} 57 \mathrm{BL} / 6$ mice. Cellular nuclei were counterstained with DAPI (blue). B: Western blot analysis of the tibialis anterior tissue from AAV-mAgrin-treated, untreated FKRP ${ }^{\mathrm{P} 448 \mathrm{~L}}$ mutant, and C57BL/6 mice with anti-agrin and anti-actin antibodies. Scale bar $=200 \mu \mathrm{m}(\mathrm{A})$. AAV, adeno-associated virus; FKRP ${ }^{\mathrm{P} 48 \mathrm{~L}}$ mutant, FKRP mutation that results in an amino acid change from proline-to-leucine at position 448; mAgrin, miniaturized form of agrin.

in all three of the muscles of the AAV-mAgrin-treated FKRP $^{\mathrm{P} 448 \mathrm{~L}}$ mice (data not shown). As expected, colocalization of agrin and laminin was restricted to the endomysium for both the untreated $\mathrm{FKRP}^{\mathrm{P} 448 \mathrm{~L}}$ mutant and C57BL/6 tissues. In addition, expression of mAgrin did not affect expression levels of laminin $\alpha 2$, laminin $\alpha 4$, or laminin a5 (Supplemental Figure S6). Immunolabeling for laminin $\alpha 2$ was associated with the muscle basement membrane and showed similar levels in all groups. Laminin $\alpha 4$ and laminin $\alpha 5$ staining was mainly concentrated around the blood vessels with little signal in the muscle basement membrane for all groups.

\section{Muscle Histopathologic/Clinical Features Are Unchanged after Treatment}

Analysis of hematoxylin and eosin sections from the AAVmAgrin-treated muscles in comparison with untreated controls demonstrated that enhanced expression of mAgrin had no beneficial impact on the dystrophic pathologic process of the FKRP ${ }^{\mathrm{P} 448 \mathrm{~L}}$ mutant mice (Figure 5A). Despite high levels of mAgrin expression 9 weeks after the initial treatment (14 weeks of age), the pathologic features of the AAV-mAgrin-treated muscles remained the same as that of the contralateral (data not shown) and untreated FKRP ${ }^{\mathrm{P} 448 \mathrm{~L}}$
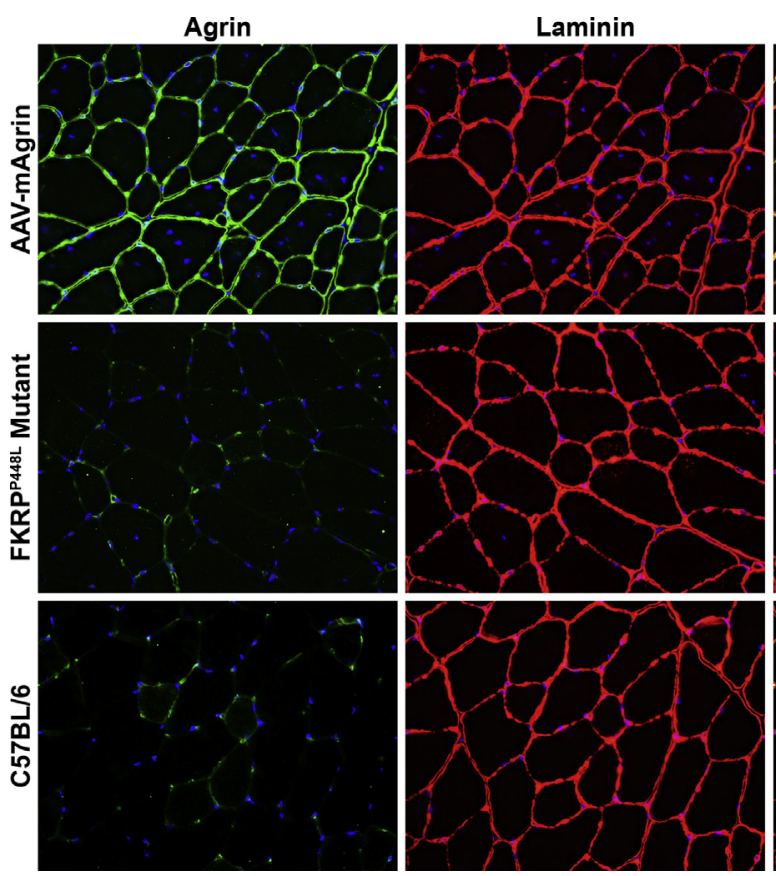

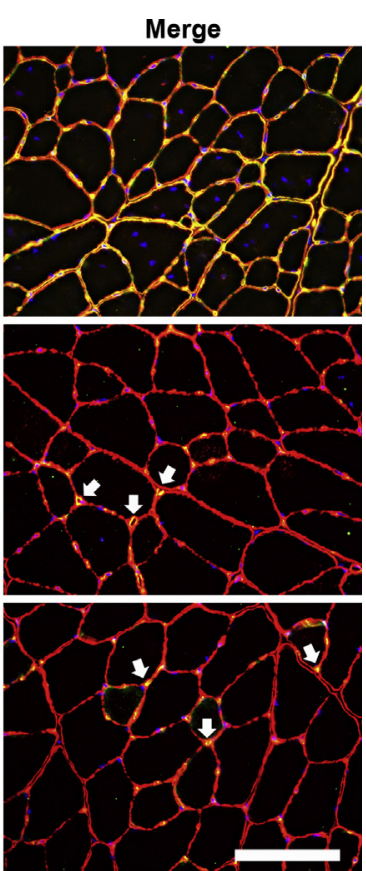

Figure 4 Agrin/laminin expression in skeletal muscle after AAV-mAgrin gene delivery. Immunohistochemical agrin (green) and laminin (red) staining of the tibialis anterior muscles from AAVmAgrin-treated, untreated FKRP ${ }^{\mathrm{P} 448 \mathrm{~L}}$ mutant, and C57BL/6 mice. Cellular nuclei were counterstained with DAPI (blue). Co-localization in the endomysium (arrowheads). Scale bar $=100 \mu \mathrm{m}$. AAV, adeno-associated virus; FKRP ${ }^{\text {P448L }}$ mutant, FKRP mutation that results in an amino acid change from proline-to-leucine at position 448; mAgrin, miniaturized form of agrin. 

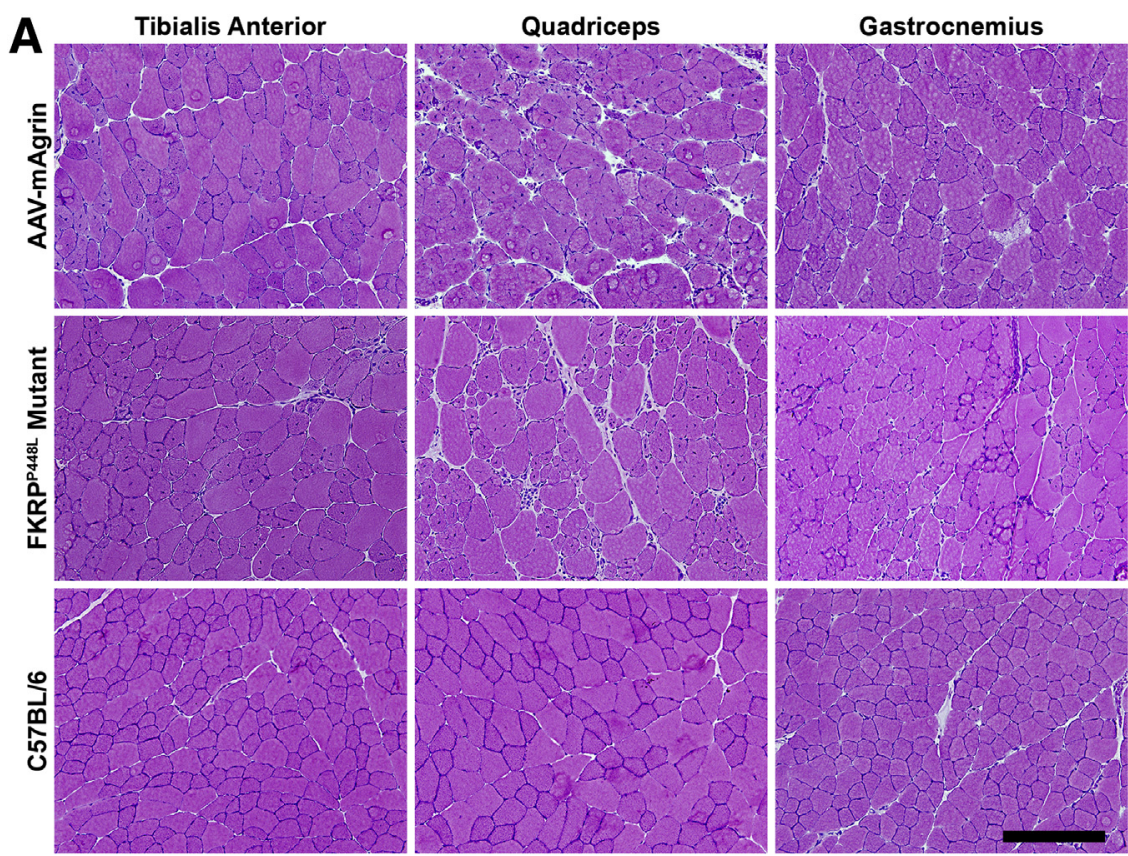

B
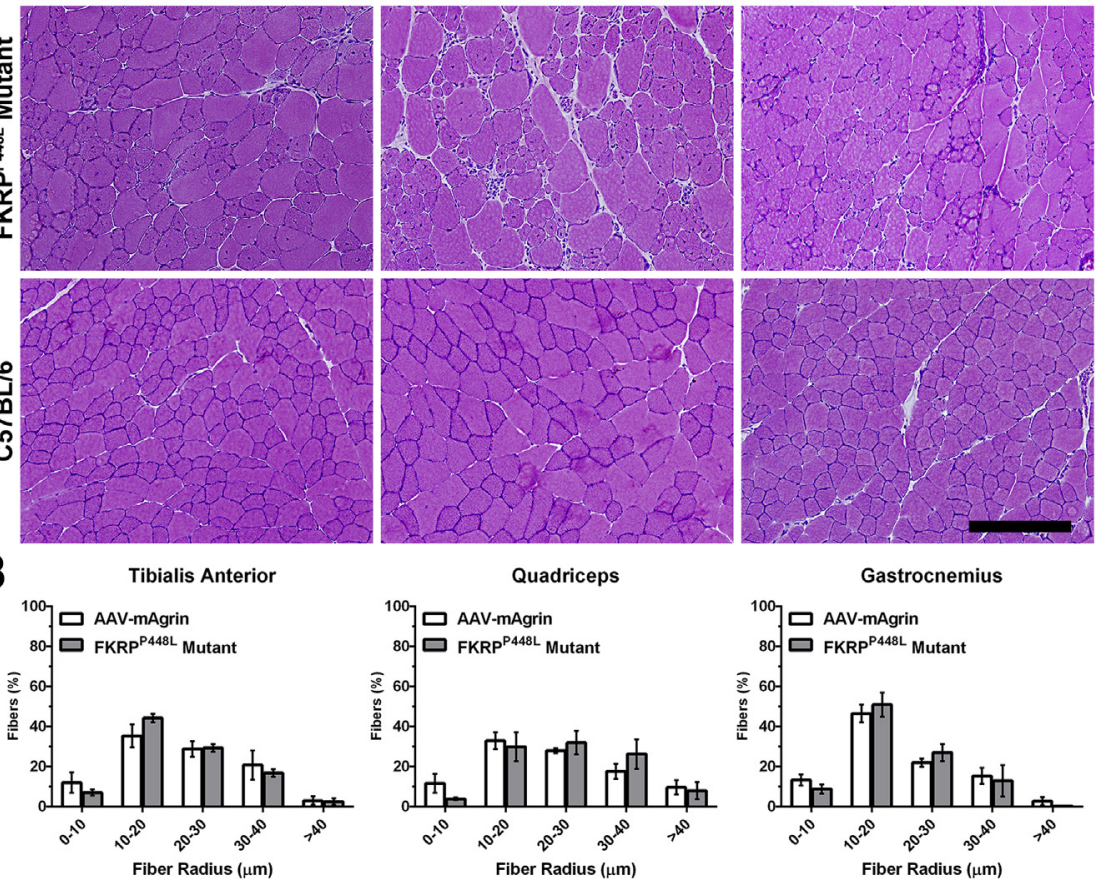

Figure 5 Histopathology of the AAV-mAgrintreated, untreated FKRP ${ }^{\mathrm{P} 448 \mathrm{~L}}$ mutant, and $\mathrm{C} 5 \mathrm{BBL} / 6$ mice. A: Hematoxylin and eosin staining of the tibialis anterior, quadriceps, and gastrocnemius muscles. B: Fiber size distribution of the tibialis anterior, quadriceps, and gastrocnemius muscles from AAV-mAgrin-treated and untreated FKRP ${ }^{\text {P448L }}$ mutant mice. The average myofiber mean equivalent radii were quantified and plotted in terms of frequency of fiber size distribution. Data are expressed as means \pm SEM. Scale bar $=200 \mu \mathrm{m}$. AAV, adeno-associated virus; FKRP ${ }^{\mathrm{P} 448 \mathrm{~L}}$ mutant, FKRP mutation that results in an amino acid change from proline-to-leucine at position 448; mAgrin, miniaturized form of agrin. mutant muscles, showing extensive degeneration/regeneration of muscle fibers. Variation of muscle fiber radii, a characteristic feature of the untreated $\mathrm{FKRP}^{\mathrm{P} 448 \mathrm{~L}}$ mutant muscles that results in hypertrophic (larger) and regenerating (small) fibers, remained unchanged in the muscles overexpressing mAgrin (Figure 5B). In addition, no difference was observed in the levels of connective tissue and collagen depositions between the AAV-mAgrin-treated and control muscles as assessed by Masson's Trichrome staining (Supplemental Figure S7).

The extent of centrally nucleated fibers was also similar between the AAV-mAgrin-treated and the untreated FKRP $^{\mathrm{P} 448 \mathrm{~L}}$ mutant mice (Figure $6 \mathrm{~A}$ ). The number of centrally nucleated fibers in the AAV-mAgrin-treated mice was $50 \% \pm 8 \%, 56 \% \pm 4 \%$, and $40 \% \pm 3 \%$ in comparison with $53 \% \pm 5 \%, 47 \% \pm 6 \%$, and $33 \% \pm 2 \%$ in the untreated FKRP $^{\mathrm{P} 448 \mathrm{~L}}$ mutant mice for the tibialis anterior, quadriceps, and gastrocnemius muscles, respectively. Serum creatine kinase activity in the AAV-mAgrin-treated mice was indistinguishable from that in the untreated $\mathrm{FKRP}^{\mathrm{P} 448 \mathrm{~L}}$ mutant mice, both of which were approximately five times higher than in C57BL/6 mice (Figure 6B). Furthermore, growth curves and muscle function tests also failed to show any significant improvement of the treated muscles in comparison with the untreated controls (Supplemental Figure S8).

\section{Discussion}

Currently, there is a lack of effective treatment options available for LGMD2I. Conventional therapies are limited to supportive care that may include physiotherapy or steroid treatment to delay muscle degeneration and orthopedic surgery to correct associated skeletal abnormalities. ${ }^{29}$ As a result, tremendous efforts have been made to develop novel therapeutic approaches. Given that LGMD2I is genetically recessive and caused by loss-of-function mutations, a gene replacement strategy is considered a highly desirable treatment option. AAV vectors have been successfully tested in a variety of dystrophic mouse models and have a proven record as being one of the most efficient vectors for gene transfer to muscles in vivo without significant immune response or toxicity. ${ }^{30-33}$ Our group and collaborators ${ }^{31,33}$ have reported that systemic administration of AAV9 expressing FKRP (AAV-FKRP) was able to effectively restore functional glycosylation of $\alpha$-DG in mouse models of LGMD2I containing human missense mutations $\left(\mathrm{FKRP}^{\mathrm{P} 448 \mathrm{~L}}\right.$ and $\mathrm{L} 276 \mathrm{I}^{\mathrm{KI}}$ ). Significant improvement in dystrophic pathology, serum creatine kinase levels, and muscle function was observed when the treatment was given at an early stage of disease progression (4-6 weeks of age). Our results provided evidence for the utility of $\mathrm{AAV}$-mediated gene 


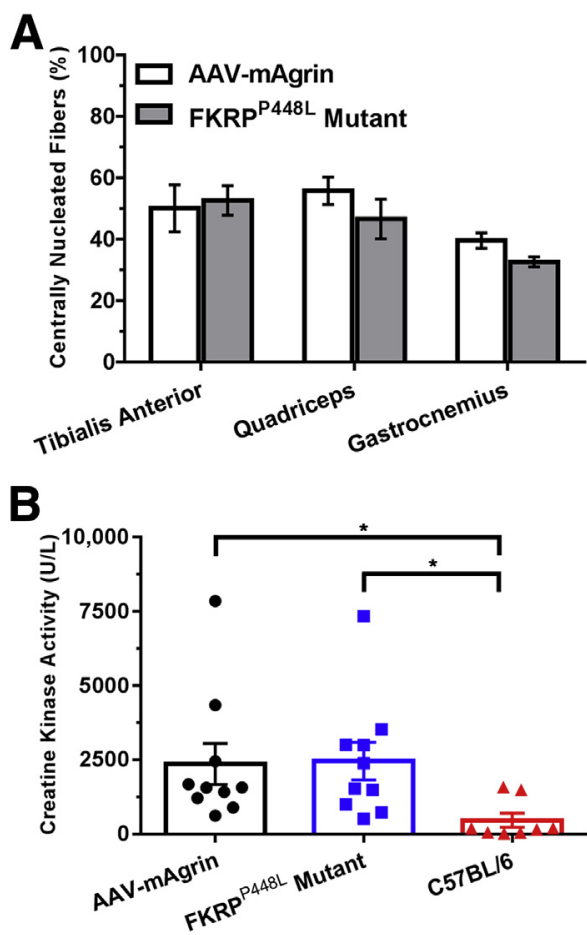

Figure 6 Quantification of muscle morphometry and creatine kinase activity. A: Percentage of centrally nucleated fibers in the tibialis anterior, quadriceps, and gastrocnemius muscles of AAV-mAgrin-treated and untreated FKRP ${ }^{\text {P448L }}$ mutant mice. B: Creatine kinase activity. AAV-mAgrin treated (black circles), untreated FKRP ${ }^{\mathrm{P} 448 \mathrm{~L}}$ mutant (blue squares), and $\mathrm{C} 57 \mathrm{BL} / 6$ (red triangles) mice. Data are expressed as means $\pm \mathrm{SEM}$. ${ }^{*} P<0.05$, statistical significance determined using an unpaired $t$-test with Welch's correction. AAV, adeno-associated virus; FKRP ${ }^{\mathrm{P} 448 \mathrm{~L}}$ mutant, FKRP mutation that results in an amino acid change from proline-to-leucine at position 448; mAgrin, miniaturized form of agrin.

replacement therapy for $F K R P$-related dystroglycanopathies. However, long-term systemic efficacy and safety of the AAV gene therapy remain to be validated in clinics, and exploring other therapeutic opportunities of treatment is essential.

Previous studies in transgenics ${ }^{12,19}$ and somatic gene therapy ${ }^{20}$ demonstrated the feasibility of rescuing the disease phenotype of laminin $\alpha 2$-deficient congenital muscular dystrophy mouse models by delivering a mAgrin muscle isoform. In general, agrin is a high-affinity binding partner of laminin(s) and dystroglycan, and the interaction of these proteins is important for the sarcolemmal integrity. ${ }^{17,18,34}$ The mechanism of action for mAgrin is that its aminoterminal region binds to laminin variants and the carboxyterminal connects to $\alpha$-DG, thereby acting as a cross-linker between the two proteins and maintaining the structural integrity of the muscle with the basement membrane. ${ }^{35}$ The $\alpha$-DG protein is highly glycosylated, and $O$-mannosylation is considered critical for interaction with extracellular matrix proteins. ${ }^{36}$ Earlier studies suggest that the lack of full glycosylation of $\alpha$-DG significantly disrupts the interactions of $\alpha$-DG with extracellular matrix proteins. ${ }^{22,37}$ These studies assessed the ligand-binding activity of agrin to hypoglycosylated $\alpha$-DG in wheat germ agglutinin-enriched lysates from muscle-eye-brain disease and Fukuyama congenital muscular dystrophy patient muscle biopsies, which showed diminished or complete lack of binding of agrin, respectively. Despite these findings, it is not clearly understood if mAgrin could bind or improve the binding of laminin(s) to the hypoglycosylated $\alpha$-DG or other membrane components in other dystroglycanopathies, such as those associated with FKRP mutations that retain residual to detectable levels of glycosylated $\alpha$-DG.

Therefore, we hypothesized that expression of mAgrin via $\mathrm{AAV}$-mediated delivery may enhance the binding of laminin(s) to residual glycosylated $\alpha$-DG and may improve the link between the laminin(s) and membrane components, including hypoglycosylated $\alpha$-DG in the dystroglycanopathy muscles, thus ameliorating dystrophic phenotypes. Our initial results in vitro showed that mAgrin significantly enhanced laminin binding to the $\mathrm{FKRP}^{\mathrm{P} 448 \mathrm{~L}}$ mutant myoblasts and fibroblasts. In some respects, this result was not anticipated based on observations that agrin binding to $\alpha$-DG highly depends on its $O$-linked mannose glycosylation. One potential explanation is that mAgrin is able to bind to hypoglycosylated $\alpha$-DG or other cell membrane proteins. Indeed, Rüegg and colleagues ${ }^{18}$ had described that agrin binds to normal muscle $\alpha$-DG with higher affinity than does laminin. Intriguingly, molar excess of mAgrin abrogated enhanced binding of laminin(s) to myoblasts and fibroblasts, resulting in the formation of larger laminin-positive aggregates, apparently bound to cells. The mechanism is not fully understood, but one possible explanation is that free agrin has a higher binding affinity to laminin(s) or cell membrane components, thus preventing the linkage formation. Despite the potential suggested by the in vitro test, our in vivo results demonstrate that expression of mAgrin was unable to alleviate the dystrophic pathologic characteristics and was insufficient at improving the overall condition of muscle function. At least two explanations can be hypothesized: most conceivably, the cell membrane components and their glycosylation are different between myoblasts/fibroblasts in vitro and myofibers in vivo, thus a lack of mAgrin binding epitopes in the dystrophic muscles is responsible for its lack of therapeutic potential in vivo; the levels of mAgrin could be critical for its effective binding to the muscle fiber membrane. The latter would be consistent with the observation that the molar excess of mAgrin relative to laminin abrogates the enhanced binding of laminin(s) to the cell surface even in myoblasts and fibroblasts with normal glycosylation of $\alpha$-DG. It is therefore possible that the levels of mAgrin expressed via AAV-mediated gene delivery are not optimal, preventing its effective binding to the fiber membrane as indicated in the cell assay. This could be tested with a series of AAV dosages expressing low-to-high levels of mAgrin; however, AAV-mediated gene delivery remains variable from muscle-to-muscle and among fibers within the same muscle. As a result, it will be difficult, if not impossible, to achieve a uniform and desirable level of mAgrin expression in body-wide muscles. Nevertheless, the defined 
co-localization of mAgrin within the basement membrane in all muscles indicates that a lack of $\alpha$-DG binding does not affect mAgrin binding to laminin(s) or other possible extracellular matrix components. This provides opportunity for further exploration of mAgrin with modification as a carrier to provide effective linkage between laminin(s) and cell membrane components, including hypoglycosylated $\alpha$ DG for potential therapeutic applications.

\section{Acknowledgments}

We thank Drs. Peter Yurchenco and Karen McKee (Robert Wood Johnson Medical School, Piscataway, NJ) for the generous gift of the purified recombinant chick mAgrin protein.

C.H.V., A.G.B., and Q.L.L. conceived experiments, acquired, analyzed, and interpreted data, and wrote/revised the manuscript; H.Z. acquired and analyzed data; C.Q. and $\mathrm{X} . \mathrm{X}$. designed and constructed the adeno-associated virus vectors. Q.L.L. is the guarantor of this work and, as such, had full access to all of the data in the study and takes responsibility for the integrity of the data and the accuracy of the data analysis.

\section{Supplemental Data}

Supplemental material for this article can be found at http://dx.doi.org/10.1016/j.ajpath.2016.09.024.

\section{References}

1. Thompson R, Straub V: Limb-girdle muscular dystrophiesinternational collaborations for translational research. Nat Rev Neurol 2016, 12:294-309

2. Brown SC, Torelli S, Brockington M, Yuva Y, Jimenez C, Feng L, Anderson L, Ugo I, Kroger S, Bushby K, Voit T, Sewry C, Muntoni F: Abnormalities in [alpha]-dystroglycan expression in MDC1C and LGMD2I muscular dystrophies. Am J Pathol 2004, 164:727-737

3. Godfrey C, Clement E, Mein R, Brockington M, Smith J, Talim B, Straub V, Robb S, Quinlivan R, Feng L, Jimenez-Mallebrera C, Mercuri E, Manzur AY, Kinali M, Torelli S, Brown SC, Sewry CA, Bushby K, Topaloglu H, North K, Abbs S, Muntoni F: Refining genotype phenotype correlations in muscular dystrophies with defective glycosylation of dystroglycan. Brain 2007, 130:2725-2735

4. Collins J, Bönnemann C: Congenital muscular dystrophies: toward molecular therapeutic interventions. Curr Neurol Neurosci Rep 2010, 10:83-91

5. Godfrey C, Foley AR, Clement E, Muntoni F: Dystroglycanopathies: coming into focus. Curr Opin Genet Dev 2011, 21:278-285

6. Chan YM, Keramaris-Vrantsis E, Lidov HG, Norton JH, Zinchenko N, Gruber HE, Thresher R, Blake DJ, Ashar J, Rosenfeld J, Lu QL: Fukutin-related protein is essential for mouse muscle, brain and eye development and mutation recapitulates the wide clinical spectrums of dystroglycanopathies. Hum Mol Genet 2010, 19:3995-4006

7. Blaeser A, Keramaris E, Chan YM, Sparks S, Cowley D, Xiao X, Lu QL: Mouse models of fukutin-related protein mutations show a wide range of disease phenotypes. Hum Genet 2013, 132:923-934

8. Whitmore C, Fernandez-Fuente M, Booler H, Parr C, Kavishwar M, Ashraf A, Lacey E, Kim J, Terry R, Ackroyd MR, Wells KE,
Muntoni F, Wells DJ, Brown SC: The transgenic expression of LARGE exacerbates the muscle phenotype of dystroglycanopathy mice. Hum Mol Genet 2014, 23:1842-1855

9. Bezakova G, Rüegg MA: New insights into the roles of agrin. Nat Rev Mol Cell Biol 2003, 4:295-308

10. Rüegg MA, Bixby JL: Agrin orchestrates synaptic differentiation at the vertebrate neuromuscular junction. Trends Neurosci 1998, 21:22-27

11. Bezakova G, Lømo T: Muscle activity and muscle agrin regulate the organization of cytoskeletal proteins and attached acetylcholine receptor (AchR) aggregates in skeletal muscle fibers. J Cell Biol 2001, 153:1453-1463

12. Moll J, Barzaghi P, Lin S, Bezakova G, Lochmuller H, Engvall E, Muller U, Rüegg MA: An agrin minigene rescues dystrophic symptoms in a mouse model for congenital muscular dystrophy. Nature 2001, 413:302-307

13. Denzer AJ, Brandenberger R, Gesemann M, Chiquet M, Rüegg MA: Agrin binds to the nerve-muscle basal lamina via laminin. J Cell Biol 1997, 137:671-683

14. Denzer AJ, Schulthess T, Fauser C, Schumacher B, Kammerer RA, Engel J, Rüegg MA: Electron microscopic structure of agrin and mapping of its binding site in laminin-1. EMBO J 1998, 17:335-343

15. Kammerer RA, Schulthess T, Landwehr R, Schumacher B, Lustig A, Yurchenco PD, Rüegg MA, Engel J, Denzer AJ: Interaction of agrin with laminin requires a coiled-coil conformation of the agrin-binding site within the laminin gamma1 chain. EMBO J 1999, 18:6762-6770

16. Ibraghimov-Beskrovnaya O, Ervasti JM, Leveille CJ, Slaughter CA, Sernett SW, Campbell KP: Primary structure of dystrophin-associated glycoproteins linking dystrophin to the extracellular matrix. Nature 1992, 355:696-702

17. Gee SH, Montanaro F, Lindenbaum MH, Carbonetto S: Dystroglycan[alpha], a dystrophin-associated glycoprotein, is a functional agrin receptor. Cell 1994, 77:675-686

18. Gesemann M, Brancaccio A, Schumacher B, Rüegg MA: Agrin is a high-affinity binding protein of dystroglycan in non-muscle tissue. J Biol Chem 1998, 273:600-605

19. Bentzinger CF, Barzaghi P, Lin S, Rüegg MA: Overexpression of mini-agrin in skeletal muscle increases muscle integrity and regenerative capacity in laminin-[alpha]2-deficient mice. FASEB J 2005, 19 : 934-942

20. Qiao C, Li J, Zhu T, Draviam R, Watkins S, Ye X, Chen C, Li J, Xiao X: Amelioration of laminin-[alpha]2-deficient congenital muscular dystrophy by somatic gene transfer of miniagrin. Proc Natl Acad Sci U S A 2005, 102:11999-12004

21. McKee KK, Capizzi S, Yurchenco PD: Scaffold-forming and adhesive contributions of synthetic laminin-binding proteins to basement membrane assembly. J Biol Chem 2009, 284:8984-8994

22. Barresi R, Michele DE, Kanagawa M, Harper HA, Dovico SA, Satz JS, Moore SA, Zhang W, Schachter H, Dumanski JP, Cohn RD, Nishino I, Campbell KP: LARGE can functionally bypass [alpha]-dystroglycan glycosylation defects in distinct congenital muscular dystrophies. Nat Med 2004, 10:696-703

23. Li J, Dressman D, Tsao YP, Sakamoto A, Hoffman EP, Xiao X: rAAV vector-mediated sarcogylcan gene transfer in a hamster model for limb girdle muscular dystrophy. Gene Ther 1999, 6:74-82

24. Xiao X, Li J, Samulski RJ: Production of high-titer recombinant adeno-associated virus vectors in the absence of helper adenovirus. J Virol 1998, 72:2224-2232

25. Snyder R, Xiao X, Samulski RJ: Current Protocols in Human Genetics. New York, Wiley, 1996

26. Awano H, Blaeser A, Keramaris E, Xu L, Tucker J, Wu B, Lu P, Lu QL: Restoration of functional glycosylation of [alpha]-dystroglycan in FKRP mutant mice is associated with muscle regeneration. Am J Pathol 2015, 185:2025-2037

27. Shigemoto K, Kubo S, Maruyama N, Yamada S, Obata K, Kikuchi K, Kondo I: Identification and characterization of $5^{\prime}$ extension of mammalian agrin cDNA, the exons and the promoter sequences. Biochim Biophys Acta 2000, 1494:170-174 
28. Zincarelli C, Soltys S, Rengo G, Rabinowitz JE: Analysis of AAV serotypes 1-9 mediated gene expression and tropism in mice after systemic injection. Mol Ther 2008, 16:1073-1080

29. Trollet C, Athanasopoulos T, Popplewell L, Malerba A, Dickson G: Gene therapy for muscular dystrophy: current progress and future prospects. Expert Opin Biol Ther 2009, 9:849-866

30. Xiao X, Li J, Samulski RJ: Efficient long-term gene transfer into muscle tissue of immunocompetent mice by adeno-associated virus vector. J Virol 1996, 70:8098-8108

31. Qiao C, Wang C-H, Zhao C, Lu P, Awano H, Xiao B, Li J, Yuan Z, Dai Y, Martin CB, Li J, Lu Q, Xiao X: Muscle and heart function restoration in a limb girdle muscular dystrophy 2I (LGMD2I) mouse model by systemic FKRP gene delivery. Mol Ther 2014, 22:1890-1899

32. Vannoy CH, Xu L, Keramaris E, Lu P, Xiao X, Lu QL: Adeno-associated virus-mediated overexpression of LARGE rescues [alpha]-dystroglycan function in dystrophic mice with mutations in the fukutin-related protein. Hum Gene Ther Methods 2014, 25:187-196
33. Xu L, Lu PJ, Wang C-H, Keramaris E, Qiao C, Xiao B, Blake DJ, Xiao X, Lu QL: Adeno-associated virus 9 mediated FKRP gene therapy restores functional glycosylation of [alpha]-dystroglycan and improves muscle functions. Mol Ther 2013, 10:1832-1840

34. Bowe MA, Deyst KA, Leszyk JD, Fallon JR: Identification and purification of an agrin receptor from Torpedo postsynaptic membranes: a heteromeric complex related to the dystroglycans. Neuron 1994, 12: $1173-1180$

35. Meinen S, Rüegg MA: Congenital muscular dystrophy: mini-agrin delivers in mice. Gene Ther 2006, 13:869-870

36. Paul TM: Congenital muscular dystrophies involving the O-mannose pathway. Curr Mol Med 2007, 7:417-425

37. Michele DE, Barresi R, Kanagawa M, Saito F, Cohn RD, Satz JS, Dollar J, Nishino I, Kelley RI, Somer H, Straub V, Mathews KD, Moore SA, Campbell KP: Post-translational disruption of dystroglycanligand interactions in congenital muscular dystrophies. Nature 2002, 418:417-422 